\title{
Continuous Spike-Wave in Slow Wave Sleep (CSWS) Mimicking Dementia in a 55-Year-Old Man with Intellectual Disability
}

\author{
Maria Arvio ${ }^{1, \dagger}$, Timo Nyrke', Oili Sauna-aho ${ }^{2}$, Nina Bjelogrlic-Laakso³
}

\begin{abstract}
Diagnostics is cumbersome in intellectually disabled patients experiencing neuropsychiatric symptoms because of overlapping disorders and also because of the age-dependent terminology. In adults conditions leading to cognitive decline are called dementia, whereas during childhood or adolescence years the resulting condition is called intellectual disability (ID) even though the causes of dementia and those of IDs do overlap. Continuous spike-wave in slow sleep syndrome (CSWSS) represents a rare childhood epileptic encephalopathy the typical EEG pattern of which is traditionally thought to resolve before the teenage. In this paper we describe a middle-aged patient with cerebral palsy and mild ID whose long-lasting neuropsychiatric symptoms were mistaken as dementia (in spite of an informative EEGfinding) until the possibility of CSWS was considered and clobazam was found to result in full recovery.
\end{abstract}

\section{Keywords:}

CSWSS, Syndrome of continuous spike-wave in slow sleep, Dementia, Epilepsy, Intellectual disability, Psychosis

\begin{abstract}
Introduction
Diagnostics in intellectually disabled patients experiencing neuropsychiatric symptoms can be cumbersome because of overlapping disorders and also because of the used terminology that is dependent on the patient's age. In adults conditions leading to memory impairment and signs of executive function are called dementia even though the term "dementia" is often wrongly used to describe a wide range of symptoms varying from memory impairment to changes in personality and mood. When cognitive decline occurs during childhood or adolescence years the resulting condition is called intellectual disability even though the causes of dementia and those of intellectual disabilities do overlap. On the
\end{abstract}

other hand, psychosis may occur at any age and people experiencing psychosis may exhibit e.g. personality changes. In other words, there may be several disorders behind highly variable neuropsychiatric symptoms making differential diagnostics challenging in this patient group.

However, there is one significant difference between adults and children experiencing a cognitive decline. The epileptic activity during brain maturation is thought to contribute to cognitive deficits resulting in intellectual disability whereas epilepsy is not generally considered as a contributing factor in dementia. The risk of epileptic seizures may be increased e.g. in Alzheimer's disease and the signs of temporal lobe epilepsy may mimic dementia

'Joint Authority for Päijät-Häme Social and Health Care, Lahti, PEDEGO Oulu University Hospital, Turku University Hospital, Finland ${ }^{2}$ Southwest Special Care Municipal Authority, Paimio, Finland

${ }^{3}$ Tampere University Hospital, Tampere, Finland

${ }^{\dagger}$ Author for correspondence: Maria Arvio, MD, PhD, Joint Authority for Päijät-Häme Social and Health Care, Lahti, PEDEGO Oulu University Hospital, Turku University Hospital, Finland, Tel. +358 29448 0000, Fax +358 8344 084; email: Maria.Arvio@phhyky.fi 
signs, but the paroxysmal nature of epileptic seizures makes epilepsy usually distinguishable from dementia. Consequently, sleep EEG is not routinely recorded when the first signs of dementia are observed in adult patients.

Continuous spike-wave in slow sleep syndrome (CSWSS) represents rare epileptic encephalopathies. One of the cornerstones in CSWSS definition has traditionally been its agerelation to childhood and resolution of the typical EEG pattern before the teenage [1]. In this paper we describe a middle-aged patient with cerebral palsy and mild intellectual disability whose long-lasting neuropsychiatric symptoms were mistaken as dementia for four years in spite of an informative EEG-finding. The consideration of CSWSS in an adult patient, and initiation of clobazam resulted in a full recovery.

\section{Case Report}

The patient has given a written permission to publish his case report. The first author has followed the patient since he was 26 years old.

Patient was born prematurely in 1955; the mother died at delivery. At the age of 8 months the patient was found to have a cerebral palsy affecting three limbs (right arm showed quite normal functions) and generalized tonic-clonic seizures. The seizures ceased on primidone treatment and two years later the medication was tapered off. The patient learned to walk and to speak fluently. At the age of six the seizures relapsed. He had complex partial seizures with an aura during which he lied down followed by head and cheek twisting. A good response was achieved with phenobarbitone and phenytoin. The patient was considered too disabled, mild intellectual disability and cerebral palsy, to attend school. However, the aunt who took care of him, organized private education and the patient learned to read fluently and tell time. At the age of 13 the complex partial seizures restarted. A left sided amydalectomy was performed due to treatment resistant epilepsy. During adolescence and young adulthood the patient had once a year a single secondarily generalized tonic-clonic seizure. In adolescence the patient passed the exam of musician and singer and besides working in shelter workshop acted as a vocalist in a choir. He had an appointment with neurologists twice a year and several wake-EEG recordings were done, all of which showed epileptiform focus on left frontotemporal area.
At the age of 44 the drug regimen consisted of phenytoin (225 mg/day) and clonazepam (4mg/ day). Brain MRI showed an almost missing right hemisphere with only some isolated patches of cortex along the skull and also the left thalamus was destroyed, left hippocampus sclerotic and white matter strongly reduced. Psychological assessment revealed mild ID; the verbal IQ was 56 and performance IQ 51 . The patient lived by himself, attending daily to cultural activities as a singer, while the cousin took care of his financial matters. At the age of 50 the patient experienced an acute cognitive decline. The cousin told that she had moved to the same house with the patient because he had become helpless and apathetic and could not tell the time of the day or remember the names of familiar people. He had become aggressive and delusional; kept on changing posture and when walking he leant on furniture. He had several "dog-seizures" (panting like a dog, tongue sticking out of mouth, walking, having tachycardia, and sweating). He had also messed with stool and urinated on a floor. B12-vitamin deficiency and dysfunction of thyroid were excluded by laboratory tests. EEG showed unilateral CSWS (Figure 1A), but his overall condition was interpreted as vascular dementia. Clonazepam was withdrawn gradually and topiramate $(100 \mathrm{mg} /$ day $)$ initiated. Special attention was paid on rehabilitation. Four years later an updated EEG had still showed unilateral, continuous, irregular fronto-central spike wave discharge, which became very prominent during sleep. Clobazam $(10 \mathrm{mg} / \mathrm{d})$ was started and soon after, all dementia, behavioral and motor symptoms disappeared. A 24-hour ambulatory EEG showed left-sided focus with slow waves, beta activity and occasionally abundant intermittent spike wave complexes, but no CSWS discharge pattern (Figure 1B). Psychological assessment was updated; the verbal IQ was 45 and performance IQ 63.

Since then he has been seizure free for over five years (clobazam $10 \mathrm{mg} / \mathrm{d}$ and topiramate $50 \mathrm{mg} / \mathrm{d}$ ); he needs help in household and financial matters, but he is otherwise quite independent and attends his daily activities.

\section{Discussion}

Based on over 50 years of clinical follow up our case suggests that CSWSS does not affect only children, but that it can manifest in adulthood as psychosis and dementia signs. The CSWSS belongs to a heterogeneous group of childhood 


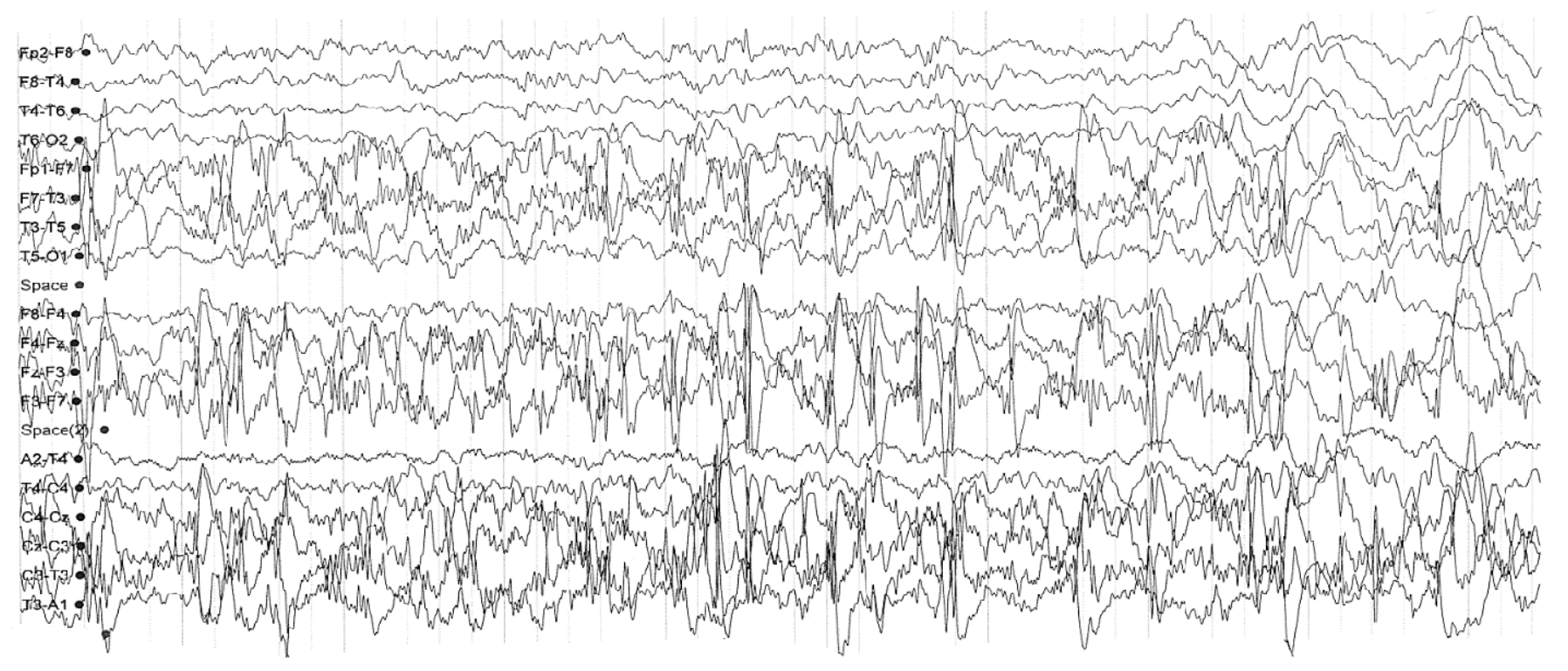

Figure 1A: The patient's EEG finding after cognitive decline and before initiation of clobazam showed continuous spike wave discharge during sleep on the left side.

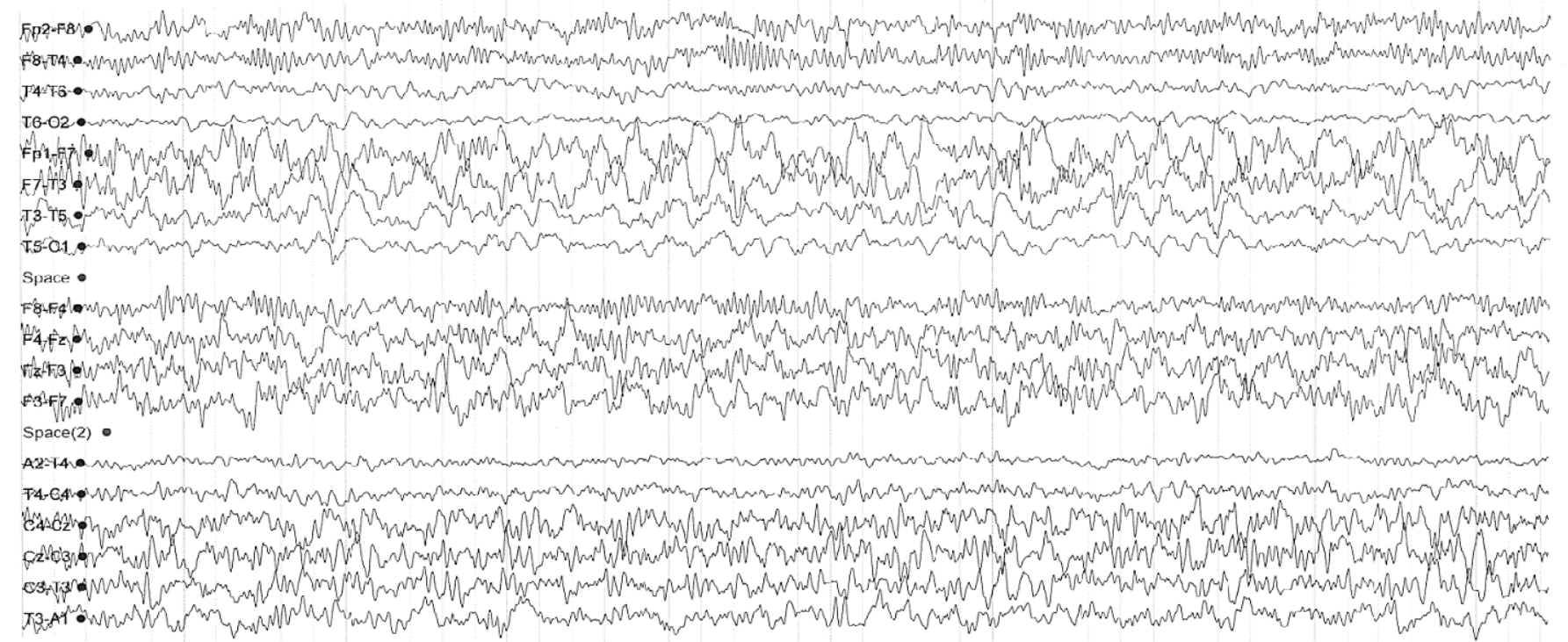

Figure 1B: TThe patient's EEG finding after the initiation of clobazam showed left-sided slow wave activity with intermixed spike wave complexes during sleep.

onset epilepsies, i.e. epileptic encephalopathies which is an electroclinically defined syndrome and often a continuum to infantile spasms, Lennox-Gastaut syndrome, structural-metabolic epilepsy or atypical benign focal epilepsy. In our patient the deterioration of general well-being and dementia/confusion at the age of 50 was found to be associated with CSWSS type EEG finding. Thus, our case challenges the prevailing understanding of CSWSS only as a childhood disorder.
Recognition of CSWSS may be extremely challenging even in pediatric patients with intellectual disability. Anamnesis may be, at most, of little help as these patients can only rarely express themselves well enough to tell of their symptoms. In addition the attacks may look atypical and be, therefore, difficult to recognize e.g. because of co-existing psychiatric and/or other somatic co-morbidities as well as because of polypharmacy. Furthermore, seizure types in CSWSS vary from atypical absences and 


\section{Research Maria Arvio}

negative myoclonias to other motor and atonic seizures. Nevertheless, some patients may show only neuropsychiatric signs and developmental decline or delay without any seizures.

CSWSS has been described already over 40 years ago, but the literature search gave only three cases where this disorder has been reported in young adults aged 19 - 21 [2-4]. Based on an EEG-finding and a good response to clobazam the CSWSS appears evident in our 55-year old patient; the somewhat atypical unilateral pattern of discharges found in our case has been described in the literature [5]. His specific epilepsy diagnosis is further supported by the facts that he had an acquired brain damage and that before the right diagnosis he had been on a narrow-spectrum antiepileptic medication both of which are known risk factors for CSWSS [6].

\section{Conclusion}

It is impossible for us to know for sure if our patient has had CSWSS episodes already in childhood because sleep-EEGs were not routinely done at that time and had not, thus, been recorded in our case either. However, it is clear that once his neuropsychiatric symptoms improved also his specific EEG-finding suggestive of CSWSS disappeared. In conclusion, our case questions the manifestation of CSWSS only in children and speaks for the clinical importance of sleep EEG-recordings in intellectually disabled adult patients, who experience treatment resistant neuropsychiatric symptoms.

\section{References}

1. Lado FA, Rubboli G, Capovilla G, et al. Pathophysiology of epileptic encephalopathies. Epilepsia 54(8), 06-13 (2013).

2. Veggiotti P, Pera MC, Teutonico F, et al. Therapy of encephalopathy with status epilepticus during sleep (ESES/CSWS syndrome): an update. Epileptic. Disord 4(1),

\section{1-11 (2012).}

3. Bensalem-Owen MK, Fakhoury TA Continuous spikes and waves during slow sleep in an adult. Epilepsy. Behav 12(1), 489491 (2008).

4. Hommet C, Billard C, Barthez MA, et al. Continuous spikes and waves during slow sleep (CSWS): outcome in adulthood. Epileptic. Disord 2(1), 107-112 (2000).
5. Fortini S, Corredera L, Pastrana AL, et al. Encephalopathy with hemi-status epilepticus during sleep or hemicontinuous spikes and waves during slow sleep syndrome: a study of 21 patients. Seizure 22(7), 565-571 (2013).

6. Mariotti $P$, Della Marca G, luvone $L$, et al. Is ESES/CSWS a strictly age-related disorder? Clin. Neurophysiol 111(3), 452-456 (2000). 